\title{
Análisis Univariante de la Competencia Digital en Educación Física: un estudio empírico Univariate Analysis of Digital Competence in Physical Education: an empirical study \\ *Francisco David Guillén-Gámez, **Maria Perrino Peña \\ *Universidad de Almería (España), **Universidad Pontificia de Salamanca (España)
}

\begin{abstract}
Resumen. La integración de las nuevas tecnologías de la información y la comunicación (TIC) en la comunidad educativa universitaria ha cambiado la manera de enseñar y comunicarse con el alumnado, propiciando la adquisición de competencias digitales. Uno de los factores clave en la integración de las TIC en contextos educativos es la formación en competencia digital del profesorado y alumnado (Castañeda, Esteve \& Adell, 2018). Sin embargo, la integración didáctica (actitud, conocimiento tecnológico y uso didáctico) de estos recursos en el proceso de enseñanza y aprendizaje en contextos universitarios no se lleva a cabo de la misma forma en todos los grados educativos ya que, en parte, depende de las especificaciones legislativas en cuanto a la utilización de las TIC en la enseñanza universitaria, especialmente en el grado en Ciencias de la Actividad Física y del Deporte (CAFYD). Por ello, los objetivos de esta investigación son: (1) conocer descriptivamente el nivel de competencia digital del alumnado universitario que estudia el grado en CAFYD; (2) conocer si existen diferencias significativas entre el conocimiento adquirido en el alumnado sobre herramientas y recursos TIC en comparación con el uso didáctico que hacen de las mismas en su enseñanza universitaria; (3) conocer si existen diferencias en el nivel de competencia digital en función del género y curso del grado educativo. Para tal fin, se ha utilizado un diseño cuantitativo no experimental por encuestas, con una muestra de 217 estudiantes del grado en CAFYD de la Universidad Pontificia de Salamanca (UPSA). Los resultados mostraron que el nivel general en competencia digital del alumnado es medio. Específicamente, las dimensiones conocimiento tecnológico y uso didáctico son percibidas con niveles medio-bajo, mientras que sus actitudes hacia las TIC sí son muy favorables. Respecto al género no fueron encontradas diferencias significativas en el nivel de competencia digital del alumnado en ninguno de los cursos del grado educativo. El nivel obtenido por los estudiantes del grado que forma a los futuros docentes de Educación Física en la etapa de enseñanza secundaria, pone entre interrogantes si estos niveles son suficientes para atender las demandas curriculares que plantea el actual sistema educativo español respaldado por la LOMCE o si, por el contrario, son niveles similares a los que puede poseer la población en general, los cuales no han recibido una formación académica y digital específica.
\end{abstract}

Palabras clave: competencia digital; TIC; Educación Física; género; estudiantes.

Abstract: The integration of the new information and communication technologies (ICT) in the university educational community has changed the way of teaching and communicating with students, promoting the acquisition of digital skills. One of the key factors in the integration of ICT in educational contexts is teachers' and students' training in digital competence (Castañeda, Esteve \& Adell, 2018). However, the didactic integration (attitude, technological knowledge, and didactic use) of these resources in the teaching and learning process in university contexts is not carried out in the same way at all educational degrees since, in part, it depends on Legislative specifications regarding the use of ICT in tertiary education, especially in the degree in Physical Activity and Sports Sciences (CAFYD). Therefore, the objectives of this research are: (1) to study descriptively the level of digital competence of university students enrolled in the bachelor of CAFYD; (2) to assess if there are significant differences between students' acquired knowledge about ICT tools and the didactic use they make of them in their university education; (3) to assess if there are differences in the level of digital competence according to gender and educational grade course. For this purpose, a non-experimental quantitative surveybased design was employed, with a sample of 217 students from the bachelor in CAFYD at the Pontifical University of Salamanca (UPSA). The results showed that students' general level of digital competence is average. Specifically, the dimensions of technological knowledge and didactic use are perceived at average-low levels, while students' attitudes towards ICT are very favorable. Regarding gender, no significant differences were found in students' level of digital competence in any of the courses of the program. The levels obtained by CAFYD students leads to an inquiry on whether these levels are sufficient to meet the curricular demands posed by the current Spanish education system supported by the LOMCE or on the contrary they are similar to those that the overall population, who has not received specific academic and digital training, may possess.

Keywords: digital competence; ICT; physical education; gender; students.

\section{Introducción}

Desde la publicación de la LOE (Ley Orgánica 2/2006 de Educación), comenzamos a incluir las denominadas Compe-

\footnotetext{
Fecha recepción: 10-04-19. Fecha de aceptación: 01-10-19

Maria Perrino Peña
}

mperrinope@upsa.es tencias Básicas como referentes de los objetivos que pretendíamos que todo ciudadano/a alcanzase a través de la educación obligatoria. Dichas competencias se clasificaron en un total de ocho (la LOMCE en 2013 las redujo a 7), dentro de las cuales la competencia digital tiene su correspondiente apartado de elevada relevancia y debe ser atendida desde todas las materias de la educación, al igual que las demás. Sin embargo, al analizar el Real Decreto 1631/2006, de 29 de 
diciembre, por el que se establecen las enseñanzas mínimas correspondientes a la Educación Secundaria Obligatoria del Estado Español (RD 1631/2006) y que desarrollan a fondo el modo en que éstas deben ser tratadas desde cada área, Atienza y Gómez-Gonzalvo (2013) advirtieron que no se hacía referencia al grado y forma en que la Educación Física ha de contribuir a la adquisición de la competencia matemática ni al tratamiento de la información y competencia digital.

Aun siendo un hecho cuanto menos sorprendente, otros autores (Palvia, Baqir \& Nemati, 2018; Ziemba, 2019; Zhou \& Purushothaman, 2019; Castells, 2000) consideran que las nuevas tecnologías de la información y la comunicación (TIC) forman parte de la cultura actual, y por ende, podríamos considerar que se han de integrar en cualquier área educativa de una manera transversal, ya que mejoran tanto el proceso de enseñanza-aprendizaje como el rendimiento académico del alumnado(Guillén-Gámez, Álvarez-García \& Rodríguez, 2018). Lo que parece claro es que la competencia debe adquirirse por todo el alumnado a través de un aprendizaje continuo, y desde la educación superior se debe formar al futuro profesorado para que posibilite estos aprendizajes en su futuro como docentes. Es más, Ortíz, Allepuz \& Sánchez (2019) destacan que el aprendizaje ha de ser continuo donde el uso de recursos TIC aparecen como pieza clave en la mejora de los procesos formativos del alumnado de Educación Física. La LOMCE (Ley Orgánica 8/2013 de Mejora de la Calidad Educativa) recoge ya la idea de que todas las competencias básicas (ahora denominadas competencias clave o $\mathrm{CV}$ ) deben ser incorporadas en mayor o menor medida a las diferentes áreas-materias descritas en el currículum, lo que obliga al profesorado a estar formado y a tratarlas en su labor educativa, y ello también incluye, por supuesto, a la materia de Educación Física (Rodríguez \& González, 2013).

Figueras, Capllonch, Blázquez, \& Monzonís (2015) en su estudio de revisión sobre la contribución de la Educación Física (EF) a las distintas competencias básicas, afirman que esta contribuye a seleccionar nuevas fuentes de información e innovaciones tecnológicas a medida que van apareciendo, en función de su utilidad para acometer tareas didácticas en los objetivos del alumnado. Del mismo modo, desde la EF, se contribuye a dominar y aplicar en distintas situaciones y contextos lenguajes específicos básicos: textual, numérico, icónico, visual, gráfico y sonoro (citando a Calvo \& Capllonch, 2013; Muñoz Díaz, 2010). Se han publicado estudios que, con carácter genérico, versan sobre percepciones del profesorado acerca del sistema competencial (Zapatero-Ayuso, González-Rivera \& Campos-Izquierdo, 2016; Hortigüela, Pérez Pueyo \& Abella, 2016) pero incidiendo en las competencias básicas o clave en su conjunto. Las percepciones concretas hacia la competencia digital y las TIC merecen un estudio particular, en este sentido, para posibilitar el avance y progreso de la enseñanza obligatoria en la etapa secundaria.

El Ministerio de Educación y Formación Profesional del Gobierno de España (MEFP, 2018) define que para que una persona sea lo suficientemente competente en materia TIC (competencia digital), requiere del conocimiento básico de las principales aplicaciones informáticas, conceptualizándolo con el saber; además, de ser capaz de utilizar eficientemente los recursos tecnológicos con el fin de desenvolverse ante los problemas en contextos reales, conceptualizándolo con el saber hacer; resaltando la importancia de tener unas actitudes activas, críticas y realistas con el fin de utilizar dichos recursos 2.0 de la manera más óptima posible, contextualizándolo en saber ser.

Multitud de autores han intentado estructurar los componentes que son necesarios para que el profesorado sea lo suficientemente competente en materia tecnológica, atendiendo al conjunto de conocimientos, habilidades y actitudes que permitan promover el aprendizaje del alumnado (Carrera \& Coiduras, 2012; Ruiz, Sánchez \& Pina, 2015; RiveraLaylle, Fernández-Morales, Guzmán-Games \& Eduardo-Pulido, 2017).

Morueta \& Gómez (2014) destacan la importancia de las actitudes, creencias e interés por las TIC como elementos clave que predicen la competencia digital del profesorado. De manera paralela, las actitudes de los docentes aparecen como un importante predictor del uso de las TIC en contextos educativos (Ayub, Bakar \& Ismail, 2015; Kretschmann, 2015; Onwuagboke \& Singh, 2016; Guillén-Gámez, MayorgaFernández \& Álvarez-García, 2018). Además, la falta de conocimientos tecnológicos y habilidades para integrar las TIC impedirá que los docentes utilicen las TIC en el aula. Esto se debe a que la actitud y el conocimiento de los profesores están correlacionados (Alagesan \& Ambikapathy, 2012; Peng \& Daud, 2016).

Sin embargo, autores como Gisbert \& Esteve (2016) o Durán, Gutiérrez \& Prendes (2016) afirman que en contextos educativos es necesario dar un paso más allá en la definición de competencia digital, ya que es necesario que el profesorado y futuros docentes conlleven una re-alfabetización. No sólo basta con que posean una correcta competencia digital, sino que han de poseer las habilidades necesarias en un contexto profesional concreto, con un buen juicio pedagógico-didáctico (Krumsvik, 2011).

Estudios como el de Rojano (2010), Pereira, Camacho \& de la Rosa (2018), Gonzalvo, Alventosa \& Devís (2018), González, Jiménez \& Moreira (2018) o Díaz, Reche, Torres \& Rodríguez (2019) han investigado y determinado la multitud de beneficios de la integración de las TIC en la Educación Física y, lo que resulta más importante, promueven un elevado interés y una mayor motivación por parte del alumnado en su proceso de aprendizaje. La mayoría de las investigaciones centradas en la competencia digital en el área de la Educación Física y el deporte ha sido estudiada a través de aplicaciones didácticas con recursos TIC (Basadre, Núñez, \& Patón, 2015; García, Castro-Lemus \& Morales, 2015; González, Sánchez-Oliva, Sánchez-Miguel, González-Ponce \& García-Calvo, 2016; Úbeda-Colomer \& Alventosa, 2016; Castro \& Gómez, 2016). Sin embargo, son muy escasas las investigaciones focalizadas tanto en las dimensiones que estructuran la competencia digital referidas al área de EF (actitud hacia las TIC, conocimiento tecnológico y uso didáctico), como en el nivel de competencia digital en función del género.

En este contexto, Barahona, García \& Pañego (2019) validaron un cuestionario para medir las actitudes e interés hacia las TIC del profesorado de Educación Física, atendiendo a la edad y al género. Con una muestra de 205 maestros de EF de centros de Educación Infantil y Primaria, los investiga- 
dores determinaron que una actitud positiva repercute en la integración de recursos TIC en la enseñanza del alumnado, revelando una correlación negativa con la variable edad. Respecto al género, no encontraron diferencias significativas en el profesorado en lo referente a sus actitudes hacia las TIC. Resultados similares fueron encontrados también por Pereira, Camacho \& de la Rosa (2018), quienes determinaron el impacto positivo del uso y actitudes hacia las TIC, como elemento alternativo ante la problemática de la no presencialidad para el desarrollo del componente físico.

En la misma línea, Romero, Castejón, López \& Fraile(2017) analizaron las percepciones en la formación inicial del alumnado del Grado de Maestro de Primaria (Mención en Educación Física) y en el Grado en Ciencias de la Actividad Física y el Deporte (CAFYD). Para ello utilizaron un total de 1.243 estudiantes universitarios españoles procedentes de la mayoría de las Comunidades Autónomas. Los resultados percibieron una mayor utilización de las TIC en el alumnado del Grado de Educación Primaria con mención en Educación Física con respecto a los estudiantes de CAFYD. Los autores tampoco encontraron diferencias significativas respecto al género. Específicamente, el alumnado de CAFYD obtuvo un valor medio en la percepción del uso de las TIC de 2.4/4.

Ramos (2020), en su estudio bibliométrico realizado en el tramo 1995-2017, puntualizó las carencias en el uso de las TIC del profesorado que imparte la asignatura de Educación Física, lo cual sin duda condicionó la actitud que finalmente manifestaban. Por ello, con el fin de integrar el uso de las TIC en los procesos de enseñanza en la EF en la etapa de Secundaria, el profesorado específico se ha de focalizar en ser facilitador de recursos, asesor y gestor del conocimiento para atender las demandas actuales de la sociedad educativa, pero, para tal fin, es necesario conocer cómo los propios profesores perciben su conocimiento en TIC, saber el uso didáctico que realizan de herramientas y recursos 2.0, y cómo es su actitud ante tal integración. Por ello, la presente investigación tiene como objetivos:

1. Conocer el nivel en competencia digital del alumnado en cada una de sus dimensiones (actitud, conocimiento y uso) acorde al curso académico del grado en CAFYD.

2. Analizar si existen diferencias significativas entre el conocimiento tecnológico del alumnado y el uso didáctico que hacen de las mismas acorde al curso académico.

3. Analizar si existen diferencias significativas en la competencia digital total del alumnado al género y curso académico.

Por lo tanto, las hipótesis sustantivas que se plantean en el estudio son:

1. El nivel de competencia digital del alumnado es medio en cada uno de los cursos

2. Existe un mayor nivel de conocimiento en herramientas TIC en comparación con la puesta en práctica de las mismas.

3. No existen diferencias en la competencia digital del alumnado en función del género.

\section{Material y método}

\section{Diseño de la investigación}

Se ha utilizado un diseño no experimental, de corte ex post facto a través de encuestas, en la cual no se ha modificado ninguna variable, sino simplemente se ha observado el problema de investigación y se ha recolectado la información necesaria para dar explicación a tal situación.

Participantes. La muestra estaba compuesta por un total de 217 estudiantes del Grado en Ciencias de la Actividad Física y del Deporte (CAFYD) de la Universidad Pontificia de Salamanca (UPSA) en el curso académico 2017/2018. De manera específica, en la tabla 1 se muestra la distribución del alumnado ateniendo al curso académico que estudia, así como al género.

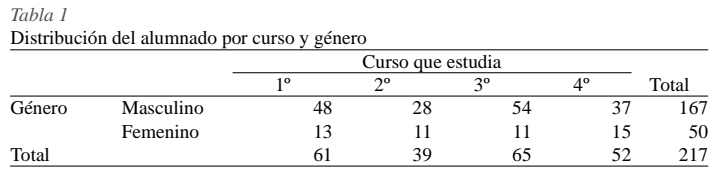

\section{Instrumento de medida}

Para medir la competencia digital del alumnado se ha utilizado el cuestionario creado por Ruiz, Sánchez \& Pina (2015). El instrumento está compuesto por 33 ítems, dividido en tres dimensiones: actitudes hacia las TIC, conocimiento en herramientas y recursos 2.0, y uso didáctico de las mismas herramientas y recursos 2.0. La dimensión actitud hacia las TIC está compuesta por 7 ítems, las dimensiones conocimiento y uso didáctico están compuesta por un total de 13 ítems cada una de ellas. La puntuación del instrumento va desde 1 a 10 puntos máximos a alcanzar.

Respecto a la fiabilidad del instrumento, se realizó a través de á de Cronbach obteniendo una alta fiabilidad con valores cercanos a uno: dimensión actitud hacia las TIC, á = 0.869; dimensión conocimiento, á = 0.859; uso didáctico, á = 0.783; total del instrumento, á $=0.894$.

\section{Análisis de los datos}

Se ha utilizado el programa estadístico SPSS V.24. Para el tratamiento de los datos, se ha llevado a cabo un análisis descriptivo del nivel de competencia digital por curso académico, así como respecto al género. El nivel de significancia establecido fue: $\mathrm{p}<0.05$, lo que significaba trabajar con un $95 \%$ de confianza y un $5 \%$ de error.

\section{Resultados}

\section{Análisis descriptivo de la competencia digital por di- mensiones}

En las siguientes tablas se muestran los resultados descriptivos en competencia digital del alumnado en cada una de las dimensiones del instrumento, en función del curso académico al que pertenece la muestra. Los datos descriptivos que se muestran son la media y su correspondiente desviación típica.

La tabla 2 muestra las actitudes hacia las TIC del alumnado. De manera general, se observa como las actitudes hacia las TIC son media-altas en cada uno de los cursos académicos, yendo ligeramente en aumento curso tras curso. De manera específica, destacar como el ítem «Es imprescindible incorporar las TIC en las aulas» es el mejor valorado por los participantes en cada uno de los cursos, siendo el ítem menos 
valorado en cada uno de los cursos «Las TIC permiten la consecución de las competencias».



En la tabla 3 se muestra los resultados descriptivos de la dimensión conocimiento tecnológico en herramientas 2.0. Teniendo en consideración el total de la dimensión, se observa como el nivel en conocimientos tecnológicos es medio en cada curso académico con un valor aproximado de cinco sobre diez. De manera específica, se observa que el mayor dominio del alumnado se encuentra en herramientas ofimáticas, espacios de interacción social como Instagram, Facebook o Twitter, así como buscadores webs. Sin embargo, herramientas más específicas como SPSS para el análisis de los datos, configuración cuestionarios o funcionamiento de bibliotecas con repositorios de documentos digitales para el trabajo académico son poco conocidas por los participantes, destacando el poco dominio de las mismas.

\begin{tabular}{|c|c|c|c|c|}
\hline \multicolumn{5}{|l|}{ Análisis descriptivo del niv } \\
\hline İtem & $1^{\circ}$ Curso & $2^{\circ}$ Curso & $3^{\circ}$ Curso & $4^{\circ}$ Curso \\
\hline Herramientas ofimáticas & $7.25 \pm 2.40$ & $8.01 \pm 1.64$ & $7.31 \pm 1.99$ & $8.08 \pm 1.45$ \\
\hline Buscadores web & $7.05 \pm 2.12$ & $7.95 \pm 1.61$ & $7.50 \pm 1.98$ & $7.79 \pm 1.90$ \\
\hline Sistemas de comunicación & $6.68 \pm 2.45$ & $7.50 \pm 1.90$ & $6.65 \pm 2.04$ & $7.21 \pm 1.90$ \\
\hline Bibliotecas digitales & $3.89 \pm 3.05$ & $5.26 \pm 2.74$ & $5.42 \pm 2.67$ & $5.53 \pm 2.39$ \\
\hline Herramientas 2.0 (blogs, wikis) & $5.94 \pm 2.67$ & $7.31 \pm 2.10$ & $6.42 \pm 2.54$ & $6.59 \pm 2.27$ \\
\hline Espacios de interacción social & $7.79 \pm 1.99$ & $7.82 \pm 2.45$ & $7.42 \pm 2.12$ & $7.64 \pm 2.45$ \\
\hline Edición de video, imagen y audio & $5.20 \pm 2.82$ & $5.64 \pm 2.35$ & $4.85 \pm 2.57$ & $5.19 \pm 2.66$ \\
\hline LMS & $5.33 \pm 2.48$ & $6.60 \pm 2.26$ & $5.31 \pm 2.95$ & $5.72 \pm 2.44$ \\
\hline Programas de análisis de datos & $3.16 \pm 2.92$ & $2.82 \pm 2.99$ & $1.96 \pm 2.40$ & $3.65 \pm 2.59$ \\
\hline Podcast & $5.08 \pm 2.89$ & $5.77 \pm 2.31$ & $4.58 \pm 2.60$ & $4.47 \pm 2.73$ \\
\hline E-porfolios & $4.18 \pm 3.12$ & $4.29 \pm 2.81$ & $3.08 \pm 2.86$ & $2.84 \pm 2.71$ \\
\hline Herramientas JClic \& Hot Potatoes & $3.03 \pm 2.89$ & $3.14 \pm 2.96$ & $1.92 \pm 2.57$ & $1.97 \pm 2.44$ \\
\hline Cuestionarios interactivos & $3.52 \pm 2.68$ & $3.72 \pm 3.08$ & $2.73 \pm 3.02$ & $3.03 \pm 2.49$ \\
\hline Media total de & $5.24 \pm 1.57$ & $5.83 \pm 1.67$ & $5.01 \pm 1.67$ & $5.36 \pm 1.49$ \\
\hline
\end{tabular}

En la tabla 4 se muestran los resultados descriptivos de la dimensión uso didáctico en herramientas 2.0. En consideración con la media total de la dimensión, se observa cómo el uso que hacen los participantes de las herramientas 2.0 es medio-bajo para cada uno de los cursos académicos. De manera específica, esto coincide con los datos mostrados en la tabla anterior (véase tabla 3) donde las herramientas ofimáticas, buscadores web o espacios de interacción social eran las más utilizadas por el alumnado, siendo el uso de programas de análisis de datos y de diseño de herramientas educativas como JClic, o bibliotecas digitales las menos utilizadas en su formación académica.

\begin{tabular}{|c|c|c|c|c|}
\hline İtem & $1^{\circ}$ Curso & $2^{\circ}$ Curso & $3^{\circ}$ Curso & $4^{\circ}$ Curso \\
\hline Herramientas ofimáticas & $7.66 \pm 2.70$ & $8.27 \pm 1.83$ & $8.54 \pm 1.76$ & $9.28 \pm 1.43$ \\
\hline Buscadores web & $7.42 \pm 2.78$ & $8.08 \pm 2.26$ & $8.50 \pm 1.81$ & $9.23 \pm 1.53$ \\
\hline Sistemas de comunicación & $5.53 \pm 3.03$ & $7.50 \pm 1.90$ & $6.46 \pm 2.72$ & $6.63 \pm 2.80$ \\
\hline Bibliotecas digitales & $3.40 \pm 2.96$ & $4.29 \pm 2.56$ & $4.35 \pm 2.47$ & $4.81 \pm 2.88$ \\
\hline Herramientas 2.0 (blogs, wikis) & $5.82 \pm 2.84$ & $5.90 \pm 2.90$ & $6.08 \pm 2.83$ & $6.39 \pm 2.54$ \\
\hline Espacios de interacción social & $7.34 \pm 2.62$ & $7.95 \pm 2.49$ & $7.42 \pm 2.86$ & $7.93 \pm 2.83$ \\
\hline Edición de video. imagen y audio & $4.88 \pm 2.87$ & $5.38 \pm 2.60$ & $3.65 \pm 2.21$ & $4.13 \pm 2.57$ \\
\hline LMS & $5.61 \pm 2.91$ & $6.79 \pm 2.29$ & $4.38 \pm 3.09$ & $5.24 \pm 2.94$ \\
\hline Programas de análisis de datos & $2.62 \pm 2.97$ & $2.50 \pm 2.69$ & $1.08 \pm 1.77$ & $3.17 \pm 2.63$ \\
\hline Podcast & $4.14 \pm 2.66$ & $4.94 \pm 2.40$ & $2.65 \pm 2.37$ & $3.56 \pm 2.77$ \\
\hline E-porfoli & $3.57 \pm 2.76$ & $3.65 \pm 2.97$ & $1.96 \pm 2.67$ & $2.12 \pm 2.49$ \\
\hline Herramientas JClic \& Hot Potatoes & $2.75 \pm 2.73$ & $2.56 \pm 2.84$ & $1.00 \pm 1.76$ & $1.20 \pm 2.01$ \\
\hline Cuestionarios interactivos & $3.03 \pm 2.63$ & $3.21 \pm 2.98$ & $1.85 \pm 2.27$ & $2.16 \pm 2.27$ \\
\hline Media total de la dimensiór & $4.91 \pm 1.52$ & $5.46 \pm 1.29$ & $4.46 \pm 1.27$ & $5.07 \pm 1.27$ \\
\hline
\end{tabular}

Análisis estadístico entre el conocimiento y uso didáctico en herramientas TIC

En la taba 5 se analiza si existen diferencias significativas entre el conocimiento adquirido por los participantes sobre las herramientas TIC que componen el instrumento ACUTIC y el uso didáctico que hacen de las mismas. El test Kolmogorov-Smirnov determinó normalidad de los datos (p. $>0.05$ ) por lo que se utilizó t-Student para muestras relacionadas para el contraste de hipótesis. Los resultados determinaron que existían diferencias significativas en el primer curso ( $\mathrm{t}=2.050$; $\mathrm{p}$. < 0.05), en el segundo curso ( $\mathrm{t}=2.445$; $\mathrm{p}$. < $0.05)$, en el tercer curso ( $\mathrm{t}=3.544 ; \mathrm{p} .<0.05)$, y no encontrando diferencias significativas en el cuarto curso del grado educativo (t=1.898; p. > 0.05).

\begin{tabular}{|c|c|c|c|c|c|c|c|}
\hline \multirow[t]{2}{*}{ Curso } & \multirow[t]{2}{*}{ Dimensión } & \multirow[t]{2}{*}{ Media } & \multicolumn{3}{|c|}{ Normalidad } & \multicolumn{2}{|c|}{ T-student } \\
\hline & & & Ks & gl & sig & $\mathrm{t}$ & Sig. \\
\hline \multirow[t]{2}{*}{$1^{\circ}$} & Conocimiento & 5.24 & 0.080 & 61 & 0.200 & 2.050 & 0.045 \\
\hline & Uso & 4.91 & 0.067 & 61 & 0.200 & & \\
\hline \multirow[t]{2}{*}{$2^{\circ}$} & Conocimiento & 5.83 & 0.119 & 39 & 0.176 & 2.445 & 0.019 \\
\hline & Uso & 5.46 & 0.150 & 39 & 0.056 & & \\
\hline \multirow[t]{2}{*}{$3^{\circ}$} & Conocimiento & 5.01 & 0.079 & 65 & 0.200 & 3.544 & 0.001 \\
\hline & Uso & 4.46 & 0.109 & 65 & 0.055 & & \\
\hline \multirow[t]{2}{*}{$4^{\circ}$} & Conocimiento & 5.36 & 0.101 & 52 & 0.200 & 1.898 & 0.063 \\
\hline & Uso & 5.07 & 0.082 & 52 & 0.200 & & \\
\hline
\end{tabular}

\section{Análisis descriptivo y estadístico acorde al género y curso académico}

En la tabla 6 se observan los resultados de cada dimensión del instrumento, así como los valores totales del mismo, acorde al género y por curso académico. Respecto a las actitudes hacia las TIC, se observa cómo el alumnado tiene actitudes medias-altas en ambos géneros para cada uno de los cursos. En lo referente al dominio de herramientas TIC, se observa que hombres y mujeres tienen un conocimiento tecnológico medio de dichas herramientas para cada uno de los cursos del grado, ocurriendo algo similar en la dimensión uso didáctico, con valores medios. Teniendo en consideración la dimensión total, se observa que los valores en competencia digital son similares en ambos géneros, no siendo en ninguno de ellos los valores altos.

\begin{tabular}{|c|c|c|c|c|c|}
\hline Curso & Género & Actitud & Conocimiento & Uso & Total Competencia Digital \\
\hline \multirow[t]{2}{*}{$1^{\circ}$} & Masculino & $7.11 \pm 1.93$ & $5.19 \pm 1.45$ & $4.76 \pm 1.49$ & $5.69 \pm 1.25$ \\
\hline & Femenino & $8.16 \pm 1.52$ & $5.41 \pm 1.99$ & $5.44 \pm 1.57$ & $6.34 \pm 1.37$ \\
\hline \multirow[t]{2}{*}{$2^{\circ}$} & Masculino & $7.79 \pm 1.59$ & $5.94 \pm 1.07$ & $5.56 \pm 1.29$ & $6.43 \pm 0.95$ \\
\hline & Femenino & $7.47 \pm 1.89$ & $5.56 \pm 1.74$ & $5.21 \pm 1.34$ & $6.08 \pm 1.43$ \\
\hline \multirow[t]{2}{*}{$3^{\circ}$} & Masculino & $7.91 \pm 1.20$ & $5.13 \pm 1.77$ & $4.50 \pm 1.31$ & $5.85 \pm 1.10$ \\
\hline & Femenino & $7.73 \pm 1.72$ & $4.44 \pm .81$ & $4.21 \pm 1.10$ & $5.46 \pm 0.82$ \\
\hline \multirow[t]{2}{*}{$4^{\circ}$} & Masculino & $7.91 \pm 1.34$ & $5.22 \pm 1.58$ & $4.85 \pm 1.30$ & $5.99 \pm 1.08$ \\
\hline & Femenino & $8.48 \pm 1.13$ & $5.72 \pm 1.24$ & $5.59 \pm 1.06$ & $6.59 \pm 0.84$ \\
\hline
\end{tabular}

En la tabla 7 se muestran los resultados al comparar estadísticamente la competencia digital total de los participantes en función del género y el curso académico. Los resultados muestran que no existen diferencias significativas en la competencia digital total del alumnado en ningún curso en función del género.

\begin{tabular}{|c|c|c|c|c|c|c|}
\hline \multirow[t]{2}{*}{ Curso } & \multirow[t]{2}{*}{ Género } & \multicolumn{3}{|c|}{ Kolmogorov-Smirnov } & \multicolumn{2}{|c|}{ t-Student } \\
\hline & & KS & $\mathrm{gl}$ & Sig. & $\mathrm{t}$ & sig \\
\hline $1^{\circ}$ & Masculino & 0.094 & 48 & 0.200 & \multirow{2}{*}{-1.636} & 0.107 \\
\hline \multirow{2}{*}{$2^{\circ}$} & Femenino & 0.269 & $\frac{13}{28}$ & 0.011 & & \multirow[b]{2}{*}{0.372} \\
\hline & Femenino & 0.128 & 11 & 0.200 & 0.903 & \\
\hline $3^{\circ}$ & Masculino & 0.085 & 54 & 0.200 & 1.105 & 0.273 \\
\hline$\overline{4^{\circ}}$ & Masculino & $\begin{array}{l}0.135 \\
0.061 \\
0.127\end{array}$ & $\begin{array}{l}11 \\
37 \\
15\end{array}$ & 0.200 & -1.937 & 0.058 \\
\hline
\end{tabular}




\section{Discusión}

Tras realizar el estudio se aprecia que, a pesar de que el alumnado posea actitudes favorables hacia el uso de las TIC en su proceso de enseñanza-aprendizaje, el alumnado posee un nivel medio-bajo de autopercepción respecto de su nivel de competencia digital en cada uno de los cursos del grado educativo, donde dicho nivel sigue siendo insuficiente para atender a las demandas educativas que se le plantean. De esta manera, se acepta la hipótesis 1 , cuyos resultados contrastan con aquellos estudios donde se afirma que el alumnado universitario posee un nivel medio en competencia digital, siendo menor del esperado (Romero et al, 2017; Ramos, 2020).

A la luz de los resultados encontrados en el alumnado acerca de sus actitudes positivas hacia las TIC, se evidencia existe un interés creciente en el alumnado del grado en CAFYD por utilizar y mejorar sus procesos de enseñanza y aprendizaje con el uso de recursos TIC, teniendo de manera similar el mismo pensamiento en cada uno de los cursos del grado educativo. Estos resultados corroboran los obtenidos por Barahona, García \& Pañego (2019) y Pereira, Camacho \& de la Rosa (2018).

Respecto a las dimensiones conocimiento tecnológico y uso didáctico, siguen poseyendo un nivel en competencia digital enfocado en herramientas digitales básicas que cualquier usuario utiliza en su vida laboral (procesadores de textos, redes sociales, buscadores web), donde la aplicación de herramientas más sofisticadas y completas, tales como programas de análisis estadísticos, bibliotecas o repositorios de documentos científicos o plataformas virtuales de aprendizaje, siguen sin estar en alza en su proceso de académico. Con respecto a su comparación, se ha encontrado que el alumnado posee mayor dominio tecnológico de los recursos TIC que el propio uso educativo que hacen de las mismas en su proceso educativo, confirmando la hipótesis 2 de nuestro estudio.

Teniendo en mente los resultados obtenidos, y siguiendo a Romero et al. (2017), se debería abandonar la premisa de que todos los estudiantes universitarios son nativos digitales y por ello se les presuponen unas elevadas capacidades en lo concerniente a las nuevas tecnologías; ni todos tienen las mismas percepciones hacia ellas, ni las mismas posibilidades, ni la misma experiencia; menos aún para su utilización como recurso didáctico provechoso en las clases de EF en la etapa de la enseñanza secundaria.

Una solución para conseguir una mayor integración de las TIC en la Educación Física, tal y como proponen Moya \& Parra (2015), sería tratar de incluir la competencia digital utilizando herramientas y recursos 2.0, utilizando el escaso tiempo de práctica motriz establecido por el currículo oficial, animando con ello a los docentes de más edad a utilizarla e integrarla en su labor educativa diaria a través de aparatos tecnológicos como wearables, recolección de datos de pruebas deportivas a través de aplicaciones web de Google +, elaboración y comunicación de los resultados a través de recursos digitales (murales, mapas conceptuales, trípticos, infografías), entre otras alternativas innovadoras. Para ello, todo proyecto o actividad elaborada debería conllevar un claro objetivo de fomento de hábitos de vida saludable y activa para la población adolescente, tanto dentro como fuera del centro escolar, debido al amplio sedentarismo que adoptan los jóvenes españoles en estas edades, especialmente las adolescentes, según los datos de las últimas encuestas sociológicas estatales sobre hábitos deportivos de los españoles (MECD, 2015).

En cuanto al género, el presente trabajo no ofrece resultados diferenciadores, al menos de manera significativa, en la percepción de la competencia digital entre alumnado del grado en CAFYD, rechazando de esta forma la hipótesis $3 \mathrm{y}$, por lo tanto, corroborando los resultados encontrados por Romero, Castejón, López \& Fraile (2017) y Barahona, García \& Pañego (2019). Si bien hay publicaciones (Gil-Juárez, Feliu, \& Vitores, 2012) que sí encuentran diferencias en el acceso, uso y control de las TIC en función del género (brecha digital de género, según el Observatorio de Igualdad de Oportunidades entre Mujeres y Hombres, 2019), cuestión que preocupa especialmente en el ámbito educativo.

Si reflexionamos en torno a la ausencia de estudios específicos sobre percepciones del alumnado de EF sobre su competencia digital entendida como el conjunto de actitudes, conocimientos tecnológicos y uso didáctico, pensamos que hemos contribuido en cierta manera al avance de esta temática. El alumnado se ve en la obligación de trabajar en la consecución de las competencias básicas plasmadas en los textos legislativos educativos vigentes en la actualidad (LOMCE, 8/2013), y necesita una apropiada (in)formación, pero requiere también de la escucha de sus necesidades, dificultades y concepciones sobre la situación.

\section{Conclusiones}

En este estudio se analiza en profundidad el nivel de competencia digital del alumnado que estudia Ciencias de la Actividad Física y el Deporte, tanto a nivel general como en relación a las dimensiones incluidas en el instrumento ACUTIC, además de identificar si existen diferencias con respecto al género del alumnado.

Es preciso destacar que, a pesar de vivir en la era de la información y la comunicación, el alumnado del grado en CAFYD no está lo suficientemente preparado en materia tecnológica para hacer frente a las demandas educativas que se les plantea en su futuro cercano, donde han de producir, difundir y consumir cultura a través de las TIC (Castellanos, Sánchez \& Calderero, 2017).

Aunque la actitud hacia las TIC del alumnado ha sido muy favorable en la investigación, se ha demostrado también que no es suficiente con una actitud predispuesta, sino que es necesario una mayor apuesta en el dominio y habilidades procedimentales de herramientas TIC para una incorporación real de dichas tecnologías en los programas académicos de la formación universitaria.

Como líneas futuras de investigación se propone la ampliación de la muestra al ámbito nacional, con el fin de poder conocer de este modo conclusiones más generalizables, como por ejemplo, muestras procedentes diferentes universidades españolas donde se imparte el grado en CAFYD; además, se debe profundizar de manera cualitativa en las percepciones sobre la propias competencias en las TIC del 
alumnado egresado, que curse el máster que le capacitará para ejercer como docente de Educación Física en el futuro.

Si bien, la principal línea de investigación futura podría ser un análisis comparativo longitudinal desde el primer curso hasta el último curso del alumnado del grado de CAFYD, con el fin de comprobar el estado y la evolución de su competencia digital durante toda su trayectoria académica, donde a través de la integración de políticas y programas educativos innovadores que utilicen recursos TIC, su nivel en competencia digital podría mejorar.

\section{Referencias}

Alagesan, A., \& Ambikapathy, L. (2012). Sikap, kemahiran dan halangan dalam penggunaan teknologi maklumat dan komunikasi bagi pengajaran bahasa tamil di sekolah menegah di Selangor, Malaysia (Doctoral dissertation, Universiti Putra Malaysia).

ANECA (2005). Libro Blanco. Título de Grado en Ciencias de la Actividad Física y del Deporte. Agencia Nacional de Evaluación de la Calidad y Acreditación. Recuperado de: http://www.aneca.es/var/media/150296/ libroblanco_deporte_def.pdf.

Atienza, R. \& Gómez-Gonzalvo, F. (2013). El Edublog. Una herramienta para la adquisición de la competencia del tratamiento de la información y competencia digital desde la Educación Física. Revista Española de Educación Física y Deportes, 402, 27-45.

Ayub, A. F. M., Bakar, K. A., \& Ismail, R. (2015, October). Factors predicting teachers' attitudes towards the use of ICT in teaching and learning. In AIP Conference Proceedings (Vol. 1682, No. 1, p. 030010- 030016). AIP Publishing.

Barahona, J. D., García, J. M., \& Pañego, M. M. (2019). Estudio de las actitudes y el interés de los docentes de primaria de educación física por las TIC en la Comunidad Valenciana. Retos: nuevas tendencias en educación física, deporte y recreación, (35), 267-272.

Basadre, R. F., Núñez, I. H. V., \& Patón, R. N. (2015). Las TIC como recurso en la didáctica de la Educación Física escolar. Propuesta práctica para la Educación Primaria. EmásF: revista digital de educación física, (35), 58-69.

Bullock, D. (2004). Moving from Theory to Practice: An Examination of the Factors that Preservice Teachers Encounter as they Attempt to Gain Experience Teaching with Technology during Field Placement Experiences. Journal of Technology and Teacher Education, 12(2), 211-237.

Carrera, X., \& Coiduras Rodríguez, J. L. (2012). Identificación de la competencia digital del profesor universitario: un estudio exploratorio en el ámbito de las Ciencias Sociales. Red-U: Revista de docencia universitaria,10 (2), 273-298.

Castañeda, L., Esteve, F., \& Adell, J. (2018). ¿Por qué es necesario repensar la competencia docente para el mundo digital? Revista de Educación a Distancia, (56), 1-20. Doi: http://dx.doi.org/10.6018/red/56/6

Castellanos Sánchez, A., Sánchez Romero, C., \& Calderero Hernández, J. F. (2017). Nuevos modelos tecnopedagógicos. Competencia digital de los alumnos universitarios. Revista electrónica de investigación edu- cativa, 19(1), 1-9.

Castro Lemus, N., \& Gómez García, I. (2016). Incorporación de los códigos QR en la Educación Física en Secundaria. Retos: Nuevas tendencias en Educación Física, Deportes y Recreación, 29, 114-119.

Díaz, I. A., Reche, M. P. C., Torres, J. M. T., \& Rodríguez, J. M. R. (2019). Impacto de las apps móviles en la actividad física: un meta-análisis (Impact of mobile apps on physical activity: A meta-analysis). Retos: nuevas tendencias en educación física, deporte y recreación, (36), 52-57.

Durán Cuartero, M., Gutiérrez Porlán, I., \& Prendes Espinosa, M. P. (2016). Certificación de la competencia TIC del profesorado universitario: Diseño y validación de un instrumento. Revista mexicana de investigación educativa, 21(69), 527-556.

Figueras, S., Capllonch, M., Blázquez, D. \& Monzonís, N. (2015). Competencias básicas y educación física: estudios e investigaciones. Apunts. Educación Física y Deportes, 123, 34-43. http://dx.doi.org/10.5672/apunts.20140983.es.(2016/1).123.04

García, I. G., Castro-Lemus, N., \& Morales, P. T. (2015). Las Flipped Classroom a través del smartphone: efectos de su experimentación en educación física secundaria. Prisma Social: revista de investigación social, (15), 296-351.

Gil-Juárez, A., Feliu, J., \& Vitores, A. (2012). Género y TIC: En torno a la brecha digital de género. Athenea Digital, 12(3), 3-9. Recuperado de: https://doi.org/10.5565/rev/athenead/ v12n3.1137

Gisbert, M., \& Esteve, F. (2016). Digital Leaners: la competencia digital de los estudiantes universitarios. La cuestión universitaria, 7 (1), 48-59.

González, J. J. P., Sánchez-Oliva, D., Sánchez-Miguel, P. A., González-Ponce, I., \& García-Calvo, T. (2016). Proyecto MÓVIL-ÍZATE: Fomento de la actividad física en escolares mediante las Apps móviles. Retos. Nuevas tendencias en Educación Física, Deporte y Recreación, (30), 38.

González, L. E. Q., Jiménez, F. J., \& Moreira, M. A. (2018). Más allá del libro de texto. La gamificación mediada con TIC como alternativa de innovación en Educación Física. Retos: nuevas tendencias en educación física, deporte y recreación, (34), 343-348.

Gonzalvo, F. G., Alventosa, J. P. M., \& Devís, J. D. (2018). Los videojuegos como materiales curriculares: una aproximación a su uso en Educación Física. Retos: nuevas tendencias en educación física, deporte y recreación, (34), 305-310.

Guillén-Gámez, F. D., Álvarez-García, F. J., \& Rodríguez, I. M. (2018). Digital tablets in the music classroom: A study about the academic performance of students in the BYOD context. Journal of Music, Technology \& Education, 11(2), 171-182. Doi: https://doi.org/10.1386/ jmte.11.2.171_1

Guillén-Gámez, F. D., Mayorga-Fernández, M. J., \& ÁlvarezGarcía, F. J. (2018). A Study on the Actual Use of Digital Competence in the Practicum of Education Degree. Technology, Knowledge and Learning, 1-18. Doi: https://doi.org/10.1007/s10758-018-9390-z

Hortigüela, D.; Pérez-Pueyo, A. \& Abella, V. (2016). ¿Cómo perciben las competencias básicas los docentes? Estudio cualitativo sobre su incorporación como herramienta de aprendizaje. Qualitative Research in Education, 5(1), 
25-48. http://dx.doi.org/10.17583/qre.2016.1348

Kretschmann, R. (2015). Physical Education Teachers' Subjective Theories about Integrating Information and Communication Technology (ICT) into Physical Education. Turkish Online Journal of Educational Technology-TOJET, 14(1), 68-96.

Krumsvik, R. J. (2011). Digital competence in the Norwegian teacher education and schools. Högre utbildning, 1(1), 39-51.

LOE (2006). Ley Orgánica 2/2006, de 3 de mayo, de Educación. Boletín Oficial del Estado (BOE), Madrid, nº 106, de 4 de mayo de 2006.

LOMCE (2013). Ley Orgánica 8/2013, de 9 de diciembre, para la mejora de la calidad educativa. Boletín Oficial del Estado (BOE), Madrid, n.295, p. 97858, de 10 dic. 2013.

MECD (2015). Ministerio de Educación, Cultura y Deporte. Encuesta de hábitos deportivos 2015. Madrid: Secretaría General Técnica, 2015.

MEFP (2018). Ministerio de Educación y Formación Profesional del Gobierno de España. Accedido el 27 de diciembre de 2018. Recuperado de: http:// www.educacionyfp.gob.es/educacion/mc/lomce/elcurriculo/curriculo-primaria-eso-bachillerato/competencias-clave/competencias-clave/digital.html

Morueta, R. T., \& Gómez, J. I. (2014). Influencias de las creencias del profesorado sobre el uso de la tecnología en el aula. Revista de educación, (363), 230-255.

Moya, E. C., \& Parra, E. A. (2015). La facilitación del el proceso de enseñanza-aprendizaje en el área de Educación Física. Web 2.0 para el alumnado de Educación Secundaria: «El rincón de la Educación Física». Revista científica electrónica de Educación y Comunicación en la Sociedad del Conocimiento, 15(2), 403-431.

Observatorio de Igualdad de Oportunidades entre Mujeres y Hombres (Instituto de la Mujer y Para la Igualdad de Oportunidades). Ministerio de la Presidencia, Relaciones con las Cortes e Igualdad. Fecha de acceso: 25 de marzo de 2019. Recuperado de: http:// www.inmujer.gob.es/observatorios/observIgualdad/ home.htm

Onwuagboke, B. B. C., \& Singh, T. K. R. (2016). Faculty attitude and use of ICT in instructional delivery in tertiary institutions in a developing nation. International Journal of Research Studies in Educational Technology, 5(1), 77-88. https://doi.org/10.5861/ijrset.2016.1428

Ortíz, D. C., Allepuz, J. P., \& Sánchez, M. L. Z. (2019). Estado actual de la Educación Física desde el punto de vista del profesorado. Propuestas de mejora. Retos: nuevas tendencias en educación física, deporte y recreación, (35), 47-53.

Palvia, P., Baqir, N., \& Nemati, H. (2018). ICT for socioeconomic development: A citizens' perspective. Information \& Management, 55(2), 160-176. Doi: https://doi.org/10.1016/j.im.2017.05.003

Peng, C. A., \& Daud, S. M. (2016). Relationship between Special Education (hearing impairment) teachers' Technological Pedagogical Content Knowledge (TPACK) and their attitudes toward ICT integration. International conference on special education in southeast asia region 6 th series 2016, p. 1-3. Seminar Antarabangsa Pendidikan Khas.

Pereira, L. G., Camacho, A. P. H., \& de la Rosa, Y. A. (2018). Las herramientas tecnológicas TIC s como elemento alterna- tiva para el desarrollo del componente físico. Retos: nuevas tendencias en educación física, deporte y recreación, (34), 222-229.

Pereira, L. G., Camacho, A. P. H., \& de la Rosa, Y. A. (2018). Las herramientas tecnológicas TIC s como elemento alternativa para el desarrollo del componente físico. Retos: nuevas tendencias en educación física, deporte y recreación, (34), 222-229.

Ramos, J. F. C. Producción científica sobre integración de TIC a la Educación Física. Estudio bibliométrico en el periodo 1995-2017 (Scientific production on the integration of ICT to Physical Education. (2020). Bibliometric study in the period 1995-2017). Retos, 37(37), 1-7.

Real Decreto 1631/2006, de 29 de diciembre, por el que se establecen las enseñanzas mínimas correspondientes a la Educación Secundaria Obligatoria del Estado Español (RD 1631/2006). BOE núm. 5, de 5 de enero de 2007, pp. 677-773 (97 págs.). Recuperado de: https://www.boe.es/ eli/es/rd/2006/12/29/1631

Rivera-Laylle, L. I., Fernández-Morales, K., Guzmán-Games, F. J., \& Eduardo-Pulido, J. (2017). La aceptación de las TIC por profesorado universitario: Conocimiento, actitud y practicidad. Revista Electrónica Educare, 21(3), 118.

Rodríguez Moneo, M., \& González Briones, E. (2013). Reflexiones sobre las competencias básicas y su relación con el currículo. Madrid: Ministerio de Educación, Cultura y Deporte.

Rojano Ortega, D. (2010). Uso de recursos TIC en la clase de Educación Física. Una experiencia positiva con el vídeo digital y el salto vertical. Retos. Nuevas tendencias en Educación Física, Deporte y Recreación, (17), 107-110.

Romero Martín, M. R., Castejón Oliva, F. J., López Pastor, V. M. \& Fraile Aranda, A. (2017). Evaluación formativa, competencias comunicativas y TIC en la formación del profesorado, Comunicar, 52, 73-82. Doi: https://doi.org/ 10.3916/C52-2017-07

Ruiz, A. B. M., Sánchez, F. A. G., \& Pina, F. H. (2015). Cuestionario para el estudio de la actitud, el conocimiento y el uso de TIC (ACUTIC) en Educación Superior. Estudio de fiabilidad y validez. Revista interuniversitaria de formación del profesorado, (83), 75-89.

Úbeda-Colomer, J. \& Alventosa, J. P. M. (2016). El blog como herramienta didáctica en educación física: la percepción del alumnado. Apunts. Educación física y deportes, 4(126), 37-45.

Zapatero-Ayuso, J. A., González-Rivera, M. D., \& CamposIzquierdo, A. (2016). Dificultades y apoyos para enseñar por competencias en Educación Física en Secundaria: un estudio cualitativo. [Difficulties and supports to teach competencies in Physical Education in Secondary: a qualitative research]. RICYDE. Revista Internacional de Ciencias del Deporte. 13(47), 5-25.

Zhou, C., \& Purushothaman, A. (2019). Developing creativity and learning design by Information and Communication Technology (ICT) in developing contexts. In Advanced Methodologies and Technologies in Artificial Intelligence, Computer Simulation, and HumanComputer Interaction (pp. 499-511). IGI global.

Ziemba, E. (2019). The contribution of ICT adoption to the sustainable information society. Journal of Computer Information Systems, 59(2), 116-126. Doi: https://doi.org/ 10.1080/08874417.2017.1312635 